\title{
The Musicality of Ethnolingustics Cognitive Benefits
}

\author{
Genc Struga*1 and Thomas Bak ${ }^{2}$ \\ ${ }^{1}$ Department of Neuroscience, University Hospital Mother Teresa, Albania \\ ${ }^{2}$ School of Philosophy, University of Edinburgh, UK
}

*Corresponding author: Genc Struga, Department of Neuroscience, University

Hospital Mother Teresa, Albania.

Received Date: January 25, 2019

Published Date: February 06, 2019

\begin{abstract}
Background: The cognitive benefits of learning native language and bilingualism project focuses on Arvanites, a bilingual population in Greece that speak Arvanitika, a dialect of Albanian language still spoken in vast areas of Greece. It is classified as a minority and an endagered language and is considered in risk of extinction. The project aims to examine possible cognitive benefits of bilingualism in native speakers of Arvanitika, including the ability for further learning and acquisition of other languages. We aim to achieve statistically important number of Arvanites equal Bilingual and monolingual to be interview using a up to date questionnare and TEA or TEA like cognitive screening.
\end{abstract}

Method: This is a cross-sectional population study including bilingual and monolingual speakers without exclusion criteria and with respects to gender equality, stratified random sampling reponders in the areas where Arvanite population traditionally lived achieving a sample number statistically important of responders in a population unofficially ranging from 200000-16000000 or $15 \%$ of population. The samples are taken from areas where traditionally there are Arvanites with more than 500 villages in different province of Greece dominantly in Epirus, Follorina, Castoria Eubea, Attica, Corinth, Boetia, Argolis, Messenia, Acheae, Peloponnese, Thraka, and settlements in Andros, Hydra, Poros, Spetsai, Salamis Participants are voluntary; they have the right to refuse participate and to withdraw their participation and data any time. To be eligible, potential participants have to be an old adult with no previous diagnosis of dementia, bilingual in Arvanitika and Greek or otherwise. The method of the population surveys is personal in-home survey with in identified areas with significant population of Arvanites with Dr. Genc Struga and supervisor CoDirector of "Bilingualisem matters" Dr. Thomas Bak in collaboration with "Bilingualsem matters" Thessaloniki Team .The team will use their Greek colleagues connections and snowball sampling approach. This method is commonly used in social sciences when investigating hard-to-reach groups. Existing subjects are asked to nominate further subjects known to them, so the sample increases in size like a rolling snowball.

Conclusion: In Albania, language characteristics and ethnography enhance the native neuroplasticity, making it easier for Albanian speaker to learn another language and to pronounce such new language with a more accurate accent. This factor is independent of CPH (critical period hypothesis) influencing L2 (second language) acquisition and with better pronunciation or accent.

Further more to benefits of speaking native language, studies have confirmed that bilinguals performed significantly better than predicted from their baseline cognitive abilities, with strongest effects on general intelligence and reading.

\section{Background}

The cognitive benefits of learning native language and bilingualism project focuses on Arvanites, a bilingual population in Greece that speak Arvanitika, a dialect of Albanian language still spoken in vast areas of Greece [1]. It is classified as a minority and an endagered language and is considered in risk of extinction[2].

The project aims to examine possible cognitive benefits of bilingualism in native speakers of Arvanitika, including the ability for further learning and acquisition of other languages [3].
Bilingualism's effect on cognitive function has been demonstrated in many studies in terms of a better performance, particularly on tests of executive functions. In parallel, neuroimaging studies showed a greater volume of frontal lobe and better structural integrity [4]. This is usually interpreted as the result of the longterm effect of switching between two or more languages. The benefits of speaking the "mother linguae" has been demonstrated also in older the subjects. Previous studies have demonstrated that 
bilingual speakers develop cognitive impairment (Mild Cognitive Impairment and dementia) up to 5 years later compared with speakers of one language [5].

Studying Albanian language has a particular importance since it represents one of the oldest surviving members of the "Balkan" and Paleo-Balkan languages, proposed as the ancestor of modern Albanian as proto-Indo-European model which is widely accepted [6]. Certain characteristics of Albanian language, such as particular words that represent a correlation of phenomena of action and sound are particularly old and believed to be ancestors of protoAlbanian. Other characteristics of Albanian language are relatively short words with the capability to form compound words or new lexemes [7].

Human speech is a well-learned, sensorimotor, and ecological behavior ideal for the study of neural processes and brain-behavior relations. Using modern neuroimaging as functional magnetic resonance imaging (fMRI), Computational Neuroscience model and DES (Direct electrical stimulation) in awake patients undergoing brain surgery, the potential for investigating neural mechanisms of speech has increased [8].

The modern view is that networking model brain processing is not conceived as the sum of several subfunctions, but results from the integration and potentiation of parallel-though partially overlapping-subnetworks. This hodotopical account improves our understanding of neuroplasticity [9].

According to the hodotopical model of speech, following the visual input, the language network is organized in parallel, segregated (even if interconnected) large-scale cortico-subcortical sub-networks underlying semantic, phonological and syntactic processing [10]. A similarity occurs when instead of a picture, a sound and a meaning is correlated as input. In the case of meaningful short words, making other compound words are compatible with this dynamic model.

Following an input that correlates sound and meaning or a lexeme composed of small, meaningful words the most important neurofunctional principal the Hebbian learning, i.e. a synaptic link between two model neurons is strengthened if both neurons are activated during the same time interval is fulfilled [11].

\section{Method}

We aim to achieve statistically important number of Arvanites equal Bilingual and monolingual to be interview using a up to date questionnare and TEA or TEA like cognitive screening ,statisical analysis of data ,publication of results and parcipation of local and international conferences and workshops. Project aims to show the possible benefit in cognitive of native speakers of Arvanitika from bilingualism, and also the ability for further learning and acquisition of other languages. This is a crosssectional population study including bilingual and monolingual speakers without exclusion criteria and with respects to gender equality ,stratified random sampling reponders in the areas where Arvanite population traditionally lived achieving a sample number statistically important of responders in a population unofficially ranging from $200000-16000000$ or $15 \%$ of population.
The samples are taken from areas where traditionally there are Arvanites with more than 500 villages in different province of Greece dominantly in Epirus, Follorina, Castoria Eubea, Attica, Corinth, Boetia, Argolis, Messenia, Acheae, Peloponnese, Thraka, and settlements in Andros, Hydra, Poros, Spetsai, Salamis[12]. Tradional settelements with significant population of Arvanites include: Central Greece: Livanates, Malesina, Martino, Phthiotis, Tanagra; Attica: Afidnes (Kiourka), Ano Liosia, Ekali, Elfsina, Kriekouki (Erythres), Kapandriti, Malakasa, Marathonas, Markopoulo, Mesogaias, Koukouvaounes (Metamorfosi), Paiania(Liopesi), Spata, Vari, Varympompi; Peloponnese: Kranidi, Methana Islands: Hydra, Spetses [12].

Participants are voluntary ; they have the right to refuse participate and to withdraw theirparticipation and data any time .They will be given an informed consent form and detailed information sheets in Greek and Arvanitika where will be described the aim of study, methods and implication of research ,risks and any discomfort that might ensues. The procedure that will be implemented in the event of unexpected or incidental findings would be clarify.

To be eligible, potential participants have to be an old adult with no previous diagnosis of dementia, bilingual in Arvanitika and Greek or otherwise. The method of the population surveys is personal in-home survey with in identified areas with significant population of Arvanites with Dr.Genc Struga and supervisor CoDirector of "Bilingualisem matters" Dr. Thomas Bak in collaboration with "Bilingualsem matters" Thessaloniki Team .The team will use their Greek colleagues connections and snowball sampling approach. This method is commonly used in social sciences when investigating hard-to-reach groups. Existing subjects are asked to nominate further subjects known tothem, so the sample increases in size like a rolling snowball [13].

A form of consent, questionnare and TEA cognitive test with be used for screening the reponders. The purpose of the study would be in comparing cognitive performance in Greek speaking only and Arvanitika -Greek bilinguals aiming the comparable the same number of monolingual Greek Arvanites with similar background. There is been a critical view of studies performed and the influence of many factors including the right methodology of studying a population group, cognitive tests used for screening, statistical analysis, environment factors, education, social economic status, cultural and political factors therefore study aim to address to this issue. The emerge in questionnaire of all factors such as healthstatus, life style, social economic level, education background, cultural and political factors including up to date promising test in cognition used in bilingualism studies.

The TEA is the only test of attention that gives a broad overview by breaking attention down into theoretically distinct factors, which can then be used as the basis of a detailed analysis of an individual's cognitive resources. These test is being used recently to many bilingual studies, interesting part of it is namely diagnosing dementia in non-dominant language in linguistic minorities [14].

The TEA consists of eight sub-tests delivered and scored using a variety of mediums. The assessment pack comes in a black 
portable carry-case which contains: one manual, which covers Standardisation, Validation, Interpretation and Administration guidelines available to be administrate also online and translated in different language with appropriate cost. A website and secure database will be developed with support of the IT of University of Edinburgh and under the supervision with already managing web site domain "Bilingualism Matters" (Bilingualism in later life, healthy ageing \& dementia)with braches in Thessaloniki, Greece.

A database of responders records will be created using SPSS software in cooperation with Statistical Department of University of Edinburgh. Study aims to address to gender dimensions and statistically importance equality between the genders in the study.

\section{Conclusion}

Special characteristics in Albanian language such as phonosymbolism or phono semantics where sound and meaning correlate, also short words compound word forming capabilities, richness in sound, make this language more suitable for brain speech processing and affecting positively the speaker abilities for learning and overall cognitive development [15].

The richness of the Albanian language is also expressed in its written form,with the large alphabet existing of 36 to 53 letters in different periods.The Albanian language has a strong ethnic tradition of singing, considered by UNESCO as a word heritage. This iso-polyphony is a form of ancient singing where voices assemble the orchestra found mainly in the south but traditionally also in north and Macedonia. It is still vividly present in modern Albania [16]. In Albania, language characteristics and ethnography enhance the native neuroplasticity,making it easier for Albanian speaker to learn another language and to pronounce such new language with a more accurate accent. This factor is independent of $\mathrm{CPH}$ (critical period hypothesis) influencing L2 (second language) acquisition better pronunciation or accent.

Other than influence of CPH on L2 speech pronunciation or foreign accent is the fact whether the $\mathrm{L} 1$ is native language and how close is this native language to L2 language etymological formation. This process is similar process seen in the musicians having a greater ability to learn and pronounce languages with a more accurate accent [16]. Further more to benefits of speaking native language, studies have confirmed that bilinguals performed significantly better than predicted from their baseline cognitive abilities, with strongest effects on general intelligence and reading [17].

A critical view is made of the Indo-European model of languages which did not take sufficient account of Albanian, the only living testimony of Pelasgic and the view of Albanians and Albanian language as the descendant of the most ancient population of Europe, the Pelasgians. This view is contested as myth by opposite the others [18-20].

The project rises the importance of learning mother language and the use scientific approach to protect the minorities rights to preserve their language and culture as describes in directives of European Parliament '(The Union)... shall respect its rich cultural and linguistic diversity, and shall ensure that Europe's cultural heritage is safeguarded and enhanced' (Treaty of European Union, Article 3). This projects brings an unique innovation where science is used in interest of people and protection of minorities. Further the project rises the importance of Arvanitika/Albanian language, one of oldest language of Europe and Indo-European languages which brings a substantial ethno linguistic values for European Community family.

\section{Acknowledgement}

None.

\section{Conflict of Interest}

No conflict of interest.

\section{References}

1. Lukas D Tsitsipis (1999) A Linguistic Anthropology of Praxis and Language Shift: Arvanitika (Albanian) and Greek.

2. Meirion Prys Jones (2013) Endangred languages and linguistic diversity in the Europian Union.

3. http://www.europarl.europa.eu/RegData/etudes/note/ join/2013/495851/IPOL-CULT_NT(2013)495851_EN.pdf.

4. Genc Struga (2018) The Cognitive Benefits of Learning Native Language and Bilingualism in a Minority Group. EC Neurology 10(2): 22-23.

5. Padilla (2016) Bilingualism in older Mexican-American immigrants is associated with higher scores on cognitive screening. BMC Geriatrics 16: 189.

6. Chertkow H, Whitehead V, Phillips N, Wolfson C, Atherton J, et al. (2010) Multilingualism (but not always bilingualism) delays the onset of Alzheimer disease: evidence from a bilingual community. Alzheimer Dis Assoc Disord 24: 118-125.

7. Millaku, Shkelqim (2018) Albanian Language is the Key of Proto-IndoEuropean Languages.

8. (2016) Why Native Albanian Speaker have an Easier Approach toward Acquisition of other Languages? J Neurol Stroke 4(5): 00153.

9. Gracco VL, Tremblay P, Pike B (2005) Imaging speech production using fMRI. Neuroimage 26(1): 294-301.

10. Duffau H (2015) Stimulation mapping of white matter tracts to study brain functional connectivity. Nat Rev Neurol 11(5): 255-226.

11. Duffau H, Moritz-Gasser S, Mandonnet E (2013) A re-examination of neural basis of language processing: Proposal of a dynamic hodotopical model from data provided by brain stimulation mapping during picture naming. Brain Lang 131: 1-10.

12. Struga G (2016) Why Native Albanian Speaker have an Easier Approach toward Acquisition of other Languages? J Neurol Stroke 4(5): 00153.

13. (2004) Encyclopedia of the World's Minorities edited by Carl Skutsch.

14. Ben-Shlomo Y, Brookes S, Hickman M (2013) Lecture Notes: Epidemiology, Evidence-based Medicine and Public Health $\left(6^{\text {th }}\right.$ ed.), Wiley-Blackwell, Oxford, USA

15. (1994) The Test of Everyday Attention (TEA) Ian H Robertson, Tony Ward, Valerie Ridgeway, Ian Nimmo-Smith Thames Valley Test Company, 1994 Reviewed by Dr. Andrew W. Mc Anespie Chartered Clinical Psychologist - Hull and East Riding Community Health NHS Trust Honorary Clinical Lecturer - The University of Hull, UK.

16. Genc Struga (2018) The Language We Speak Affects Our Cognition. EC Neurology 10(4): 342.

17. Genc Struga (2018) How the Musicality of Ethnolinguistic Affects the Cognitive Abilities for Further Learning. Arch Neurol \& Neurosci 1(1).

18. Bak TH, Nissan J, Allerhand M, Deary IJ (2014) Does Bilingualism Influence Cognitive Ageing? Annals of Neurology 75(6): 959-963. 
19. Rapper G (2009) Pelasgic Encounters in the Greek-Albanian Borderland Border Dynamics and Reversion to Ancient Past in Southern Albania. Anthropological Journal of European Cultures: 50-56.
20. Bilingualism Matters opened in Thessaloniki, Greece, in January 2012, under the name Me 2 glosses. 